\title{
Effects of Securigera Securidaca Seed Extract, Alone and in combination with Glibenclamide, on Circulating Levels of Pro-Angiogenic/Anti- Angiogenic Biomarkers in Hyperglycemic Rats
}

\section{Shahin Alizadeh-Fanalou}

Iran University of Medical Sciences

Mohammad Babaei

Bu Ali Sina University

Elham Bahreini ( $\sim$ bahreini.e@iums.ac.ir)

Iran University of Medical Sciences

Short report

Keywords: Angiogenesis, Diabetes, Glibenclamide, Securigera securidaca, TGF- $\beta$, VEGF

Posted Date: August 5th, 2020

DOI: https://doi.org/10.21203/rs.3.rs-52508/v1

License: (c) (1) This work is licensed under a Creative Commons Attribution 4.0 International License.

Read Full License 


\section{Abstract}

Background: Many diabetic patients use herbal medicines in addition to their mainstream treatments. Plants contain a well-known and unknown set of compounds that may exacerbate or improve diabetes complications. Thus, the side effects of these herbs should be known before prescribing. The aim of the study is to investigate the effects of hydroalcoholic extract of Securigera securidaca (L.) Degen \& Dorfl (S. securidaca) seed (HESS) on angiogenesis/anti-angiogenesis balance in Streptozotocin (STZ)-induced diabetic rats, alone and in combination with glibenclamide.

Methods: The groups involved in this animal study included diabetic and healthy control groups, groups treated with three doses of HESS, group treated with glibenclamide, and groups received combination therapy. Serum samples were taken and analyzed for the levels of angiogenic/ anti-angiogenic biomarkers.

Results: Induction of diabetes increased serum levels of angiogenic agents and decreased circulating anti-angiogenic factors. The herbal extract, even with the highest dose, had little effects on the blood levels of the tested biomarkers except with TGF- $\beta$. Glibenclamide was more effective than the highest dose of HESS in preventing the increase in serum levels of angiogenic factors and in inhibiting the decrease in anti-angiogenic agents in diabetic rats. Combination therapy with the highest dose of HESS partly enhanced the glibenclamide effects.

Conclusions: Although glibenclamide was more effective than the highest dose of HESS used in this study in preventing changes in serum concentrations of angiogenic/ anti-angiogenic biomarkers in the diabetic animals, this study show that $S$. securidaca has no side effects on diabetes complications caused by vascular disorders and neovascularization, and still it can be used as a herbal supplement with the standard drug.

\section{Background}

Vascular disorders are the main cause of myocardial infarction, renal failure, blindness, and impaired wound healing in diabetes. These complications raised from progressive disorders in small, medium, and large arteries, increase the risk of peripheral ischemia. Normal tissue reaction to restore its function and minimize ischemic injury is the formation of new vessels from large arteries. Still, diabetic wound healing and myocardial complications are exceptions from the rule(1). However, firm evidence suggests that angiogenesis is affected by a counterbalance between angiogenic and anti-angiogenic stimuli, which may be influenced by a variety of herbal or synthetic drugs used by diabetic patients.

Increased oxygen consumption due to a shift toward fatty acid utilization and also the production of reactive oxygen species (ROS) associates with a decrease in cellular oxygen availability and hypoxia (2). Hypoxia and ROS are the main stimulators of hypoxia-inducible factors (HIFs) by influence on HIF-1a subunit stability. In normoxia, prolyl hydroxylase hydroxylates HIF-1a and causes it to be degraded rapidly, whereas hypoxia and ROS block this process $(3,4)$. Stabilized HIF-1 a bond to HIF-1 $\beta$ activates 
transcription of several genes for adaptation to hypoxia and ischemia, including pyruvate dehydrogenase kinase 1 to maintain anaerobic glycolysis, VEGF and VEGF receptor to promote angiogenesis and other adaptive processes to accurate tissue alterations. In addition to hypoxia and ROS, HIF-1 a expression can be induced by numerous other factors, including nitric oxide (NO), inflammatory cytokines such as TNF-a, pro-inflammatory factors such as hormone-like growth factors such as TGF- $\beta$ (5). As reported by some researches $(6,7)$, HIF-1 a expression may also be reduced by FGF21, a multifunctional factor with the protective effects on diabetic complications and energy hemostasis (8). VEGF is also up-regulated upon stimulation of such factors. The persistent elevation of HIF-1 a level in diabetes and overexpression of VEGF involved in abnormal angiogenesis may cause tissue injuries such as renal failure and some ocular diseases. Despite this phenomenon, a reduction in the expression of myocardial HIF-1a and VEGF exists during diabetic cardiomyopathy, which decreases the capillary density, increases fibrosis, and lowers contractility. Also, studies on excisional skin wounds and fibroblasts of $d b / d b$ mice showed a marked reduction in HIF-1 a expression and Akt/HIF-1a axis activity $(5,9)$. The crucial effect of VEGF on vascular remodeling and collateral formation, are mediated by binding to its receptors, particularly FLT-1 and FLK-1 which are upregulated via HIF-1a. This binding is typically prevented by the natural circulating form of FLT-1 (sFLT-1). sFLT-1 is another spliced variant of FLT-1 that acts as a VEGF antagonist and a potent inhibitor of angiogenesis. Therefore, altered plasma levels of VEGF and SFLT-1 may be linked to the severity of diabetic vascular complications. It has been demonstrated that the FLT-1 receptor is essential in endothelial cell-cell or cell-matrix interactions, while the FLK-1 receptor regulates differentiating endothelial cells and mitogenesis(10).

Plant phenolic or polyphenolic compounds as natural secondary metabolites have been considered by researchers due to their medicinal properties including antioxidant, anti-inflammatory, antimicrobial, pro/anti-angiogenic, antidiabetic, cardioprotective, hepatoprotective and neuroprotective activities (11, 12). Polyphenolics divided into flavonoids and non-flavonoids are benzene derivatives with carboxylic, hydroxyl, and/or methoxyl groups (13). Several studies have demonstrated both anti- and proangiogenic properties of such phytochemicals with the possible effects on involved factors such as VEGF, HIF-1a, FGF21, TGF, and inflammatory factors (13-15).

Securigera securidaca (L.) Degen \& Dorfl (S. securidaca), with local names of Adasol-molk and Bitterlentils, belongs to the Fabaceae family. The seed of the plant is used in Iranian folk medicine and also by Egyptians and Indians since ancient times to treat several ailments such as diabetes, hyperlipidemia, and hypertension $(8,16,17)$. In a chain study, we examine the positive or negative side effects of $S$. securidaca on diabetes complications. Our previous experiment showed that the herbal extract as a supplement could improve the hypoglycemic, antioxidant, and anti-inflammatory properties of the standard drug (8). In this short report, the possible effect of $S$. securidaca seeds on neovascularization was evaluated in the diabetic animal model, alone and in combination with glibenclamide.

\section{Methods}

Seed extract preparation and determination of total phenolic and flavonoid content 
As mentioned, the current study is a continuation of our previous studies on the side effects of $S$. securidaca seed by predicting its potential to prevent angiogenesis. S. securidaca seed (herbarium code of PMP-756) extraction and determination of total phenolic and flavonoid contents have been described in detail in our previous publication (8). Briefly, the ground seed was extracted with $70 \%$ ethanol and concentrated by rotary evaporation. The total phenolic content of the hydroalcoholic extract was determined using the Folin Ciocalteau reagent according to the method of Singleton and Rossi(18). The total flavonoid content of the crude extract was estimated using the aluminum chloride colorimetric method and quercetin as a standard(19).

\section{Experimental animals}

Diabetes was induced in male Wistar rats (233-247g) by intraperitoneal injection of STZ ( $55 \mathrm{mg} / \mathrm{kg}-\mathrm{BW})$, and rats with blood sugar above $250 \mathrm{mg} / \mathrm{dL}$ were considered as diabetic model.

\section{Experimental design}

Three doses of HESS, 100, 200, and $400 \mathrm{mg} / \mathrm{kg}-\mathrm{BW}$ (17) and one dose of glibenclamide ( $5 \mathrm{mg} / \mathrm{kg}$-BW) (20) were considered for administration. Experimental groups were group I as healthy control (NC), group II as diabetic control (DC), groups III to V as diabetic rats treated with HESS doses of 100 (E-100), 200 (E200) and $400(\mathrm{E}-400) \mathrm{mg} / \mathrm{kg}-\mathrm{BW}$, respectively, group $\mathrm{VI}$ treated with glibenclamide (G) $5 \mathrm{mg} / \mathrm{kg}-\mathrm{BW}$ and groups VII and VIII treated with both G and HESS (200 and $400 \mathrm{mg} / \mathrm{kg}-\mathrm{BW}$ ) as G+HESS-200 and G+HESS400 groups, respectively. The animals were gavaged for 35 consecutive days. At the end of the study, the rats were anesthetized with chloroform. The blood sample was collected by cardiac puncture, centrifuged at $3000 \mathrm{rpm}$ for $10 \mathrm{~min}$, and serum was stored at $-20^{\circ} \mathrm{C}$ until the use.

\section{Biochemical analysis}

Serum Insulin (MBS724709), VEGF (MBS843480), TGF- $\beta$ (MBS260302), FGF21 (MBS2024083), sFLT-1 (MBS2602003), and FLK (MBS282637) were assayed using Rat ELISA Kits from MyBioSource Company and multi-plate ELISA reader (ELISA Reader-DANA-320., Japan). Detection of total NO was performed spectrophotometrically using rat nitric oxide assay Kit (ZellBio GmbH, Germany, a lot: ZB-NO-48A). NO has a very short half-life, so it was quantified by its metabolites (nitrite and nitrate) based on the Griess $\operatorname{method}(21)$.

\section{Statistical analysis}

The normality of the data was verified by Shapiro-Wilk using SPSS software, version 25 (IBM Corp., Armonk, N.Y., USA). Then one way-ANOVA followed by Tukey's test was used to determine differences between individual groups. The data were expressed as means \pm standard deviation (SD). Association between parameters was evaluated by Simple Linear Regression. The significance level was set at $\mathrm{P}<0.05$. 


\section{Results}

Figure 1 indicates antiparallel changes in serum insulin and glucose among study groups (ANOVA: $P<0.001)$. HESS significantly and dose-dependently increased insulin levels and decreased serum glucose concentrations in diabetic rats $(P<0.05)$. Glibenclamide was more effective than the highest dose of HESS in this regard $(P<0.05)$, and its combination with HESS partly enhanced its effect on increasing serum insulin and decreasing serum glucose levels of diabetic rats $(P>0.05)$.

Figure1: Comparison of Insulin (A) and glucose (B) levels among experimental and control groups. HESS (hydroalcoholic extract of $S$. securidaca seeds); G (glibenclamide); DC (Diabetic control); NC (normal control. (Values are means \pm SD.

* Significant difference degree among groups.

Figure 2 shows changes in serum levels of angiogenic/ anti-angiogenic biomarkers in control and treated groups. One-way ANOVA revealed significant differences in the serum levels of VEGF, FLK-1, sFLT-1, FGF21, and TGF- $\beta(P<0.01)$ between study groups. In the DC group, the serum concentrations of VEGF and FGF21, and TGF- $\beta$ were significantly increased $(P<0.01)$, while serum levels of FLK-1 and sFLT-1 were significantly decreased $(P<0.01)$ compared to healthy controls. The results showed that treatment with different doses of HESS slightly altered serum levels of VEGF, sFLT-1, FLK-1 and TGF- $\beta$ in a dosedependent manner in diabetic rats, but only reduced TGF- $\beta$ levels in treatment with the highest dose of HESS (400mg $/ \mathrm{kg}-\mathrm{BW}$ ) became statistically significant compared to DC and two lower doses of HESS $(P<0.01)$. Administration of HESS resulted in a dose-dependent reduction in circulating levels of FGF21, but this reduction was not statistically significant compared to the $D C$ group $(P>0.05)$. Compared to the highest dose of HESS, glibenclamide significantly decreased the serum levels of VEGF and TGF- $\beta$ $(P<0.01)$, and non-significantly increased the serum levels of FGF-21, FLK-1 and sFLT-1 $(P>0.05)$ in diabetic rats; however, except with FGF-21, the levels of the mentioned parameters were still differ from those in the healthy group $(P<0.05)$.

According to the data, it seems that combinatorial therapy with glibenclamide and the highest dose of HESS partially enhanced the effects of glibenclamide on reducing the concentrations of angiogenic factors (VEGF, FGF21, and TGF- $\beta$ ) and increasing the levels of antiangiogenic agents (FLK-1 and sFLT-1), but these improved effects were not statistically significant $(P>0.05)$ except with TGF- $\beta(P=0.02)$. However, combination therapy altered the concentrations of FGF21, sFLT1 and FLK-1 to a comparable extent to the NC group ( $P>0.05)$. NO levels significantly increased in the DC group compared to the NC group $(P<0.05)$. Administration of the highest dose of HESS could effectively reduce NO production in diabetic rats $(P<0.05)$ and concomitant use with glibenclamide further reduced NO levels to a comparable extent in the NC group $(P>0.05)$. The effect of glibenclamide alone on the NO levels was comparable to the middle dose of HESS $(200 \mathrm{mg} / \mathrm{kg}-\mathrm{BW})(P>0.05)$.

Figure2: comparison of pro/ anti-angiogenic factors levels among experimental and control groups. (A) VEGF (Vascular endothelial growth factor), (B) sFLT-1 (soluble fms-like tyrosine kinase), (C) FLK-1 (fetal 
liver kinase 1), (D) FGF21 (fibroblast growth factor 21), (E) TGF- $\beta$ (transforming growth factor-beta), (F) NO (nitric oxide). HESS (hydroalcoholic extract of $S$. securidaca seeds); G (glibenclamide); DC (Diabetic control); NC (normal control.(Values are means \pm SD.

* Significant difference degree among groups.

\section{Correlations between VEGF levels and other studied biomarkers}

Regression analysis indicated (table 1 ) that VEGF levels were positively correlated with the parameters of TGF- $\beta$, FGF21, NO, and TNF- $\alpha(P<0.001)$, and negatively correlated with FLK-1 and sFLT-1 $(P<0.001)$.

Table1. Correlation matrix of the study variables

\begin{tabular}{|llllll|}
\hline & 1 & 2 & 3 & 4 & 5 \\
\hline 1. VEGF & & & & & \\
\hline 2. TGF- $\beta$ & $0.897^{\star}$ & & & & \\
\hline 3. FGF-21 & $0.760^{*}$ & $0.847^{\star}$ & & & \\
\hline 4. FLK-1 & $-0.864^{*}$ & $-0.880^{*}$ & $-0.694^{*}$ & & \\
\hline 5. SFLT-1 & $-0.844^{*}$ & $-0.858^{*}$ & $-0.699^{*}$ & $0.773^{*}$ & \\
\hline 6. NO & $0.671^{*}$ & $0.709^{\star}$ & $0.639^{*}$ & $-0.717^{*}$ & $-0.658^{\star}$ \\
\hline
\end{tabular}

*. Correlation is significant at the 0.01 level.

\section{Discussion}

S. securidaca is widely used in Iranian folk medicine to treat diabetics along with the mainstream treatments (herbal and standard drug combination). S. securidaca, like other plants, contains a wellknown and unknown set of compounds that may have positive or negative side effects. In our previous study, antidiabetic and antioxidant properties of hydroalcoholic seed extract of $S$. securidaca were evaluated in streptozotocin-induced diabetic rats, and the results were compared with the effects of the standard drug of glibenclamide, alone and in combination with HESS(8). We experienced that HESS reduced blood sugar and insulin resistance dose-dependently, and the effect was enhanced when used in combination with glibenclamide. HESS was more effective than glibenclamide in lowering the body oxidative state and pro-inflammatory cytokines such as hs-CRP and TNF-a, and raising antioxidant capacity. In the present short study, we assessed the possible effects of HESS on angiogenesis as the most critical process in the occurrence of diabetes complications and compared the results with the effects of glibenclamide. 
In agreement with the previous studies, induction of diabetes increased circulating levels of VEGF, FGF21, and TGF- $\beta$ and decreased plasma concentrations of FLK-1 and sFLT-1 in STZ-treated rats $(22,23)$. In addition to the effect on its secreted tissue as a paracrine factor, VEGF enters circulation and can exert its effects on other tissues that express high levels of its receptors. Therefore, lowering blood levels of VEGF could be considered as a therapeutic target. Circulating FLK-1 and sFLT-1 as natural VEGF attenuators can attache to VEGF and neutralize it(24). Administration of HESS did not show a protective effect in preventing an increase in serum VEGF concentration and a decrease in its soluble receptors, FLK-1 and sFLT-1 in diabetic rats. While HESS is rich in phenolic and flavonoid compounds $(93.3 \pm 1.5 \mathrm{mg} \mathrm{GAE} / \mathrm{g}$ (DW) and $46 \pm 1.7 \mathrm{mg} \mathrm{QE} \mathrm{/g} \mathrm{(DW),} \mathrm{respectively)(8),} \mathrm{these} \mathrm{findings} \mathrm{are} \mathrm{inconsistent} \mathrm{with} \mathrm{the} \mathrm{results} \mathrm{of}$ some studies that have stated that polyphenolic compounds downregulate the expression of angiogenic factors as an index of neovascularization $(25,26)$. Park et al. reported that polyphenols prevented the hypoxia-induced angiogenesis by reducing HIF-a transcriptional activity without altering its mRNA level, leading to reduce the expression of HIF-a downstream targets, including VEGF (27). Cerezo et al. showed that VEGF-receptors were potently inhibited by some specific polyphenols(28). They stated the polyphenols appeared to be attached to the similar region of VEGF involved in the interaction with its receptor, and the potent inhibitory was proportional to the specificity and binding affinities. Although HESS dose of $400 \mathrm{mg} / \mathrm{kg}$.BW was a sufficient amount to lower blood sugar and prevent from oxidative stress caused by hyperglycemia, it may be a low dose in modulating the factors involved in angiogenesis. In this regard, glibenclamide was more effective than the highest dose of HESS in preventing an increase in serum VEGF levels and a decrease in circulating FLK-1 and sFLT-1 in diabetic rats. Serum levels of FLK1 and sFLT-1 in diabetic group trated with both glibenclamide and E-400 were relatively comparable to those in healthy rats, but there was still a significant difference in VEGF values. Kimura et al., in their study on sulphonylureas treatment and diabetic retinopathy, reported that glibenclamide could not downregulateVEGF and inhibit ischemia-induced retinal neovascularization(29). Therefore, according to our results, it can be claimed that glibenclamide may reduce blood VEGF via the upregulation of circulating FLK-1 and sFLT-1 and the combination therapy with the highest dose of HESS partly enhanced its protective effect.

The highest dose of HESS effectively prevented the increase in NO levels in diabetic rats, while glibenclamide had little effect in this regard. Combination therapy with glibenclamide partially enhanced the effect of HESS in reducing NO levels. Recent studies have shown that NO is involved in the activation of HIF-1 a through a nonhypoxic pathway that leads to VEGF upregulation (30). Our results also showed such correlation among the levels of NO and VEGF.

The highest dose of HESS and glibenclamide showed inhibitory effects on increasing serum TGF- $\beta$ levels in diabetic rats, and their combined administration enhanced the effect of each on reducing serum TGF- $\beta$ concentrations to the comparable extent with the levels in non-diabetic rats. Upregulation of TGF- $\beta$ in long-term hypoglycemic condition plays an important role in neovascularization by stimulating the expression of angiogenic factors such as VEGF and pro-inflammatory cytokines such as TNF-a (31). It is a pro-fibrotic mediator that enhances the formation of extracellular matrix in fibrotic diseases like retinopathy and nephropathy. 
FGF21 is a multifunctional factor that can be produced in the liver under hyperglycemic conditions as a compensatory mechanism for maintaining metabolic homeostasis. In these conditions, it stimulates insulin secretion and reduces insulin resistance and acts as a hypoglycemic, hypolipidemic, ketogenic, and antioxidant inducer agent(32). Correction of hyperglycemia by injecting insulin, standard drugs, or herbal medicine can lower serum FGF21 levels $(8,33)$. Lin et al., in their study on the effects of FGF21 on renal fibrosis, reported that FGF21 prevents renal fibrosis via the downregulation of the TGF- $\beta$ (34). However, no direct effect of FGF21 on VEGF expression has been reported so far. Here, despite high levels of FGF21 in diabetic control rats, elevated levels of VEGF and TGF- $\beta$ may be due to several factors contributing to increased production of angiogenic factors in uncontrolled diabetes, and FGF21 alone may not be sufficient in prevention. This relationship is justified via the positive correlation of FGF21 levels with angiogenic factors in the regression results. In this regard, glibenclamide decreased TGF- $\beta$ and FGF21 levels in diabetic rats more effectively than the highest dose of HESS, and combination therapy with the highest dose of HESS partially enhanced the effectiveness of glibenclamide. FGF21 exerts parts of its role in modulating energy hemostasis via the increase in circulating adiponectin, which now approved that suppresses retinal and choroidal ocular neovascularization. Adiponectin also suppresses TNF-a transcription through mRNA instability, which results in a decrease in the retinal and choroidal neovascularization (35).

\section{Conclusions}

Taking together, our results indicated positive correlations among angiogenic promoting factors $(P<0.001)$ and also among antiangiogenic factors $(P<0.001)$, and a negative relationship between these two sets of parameters $(P<0.001)$. Diabetes was associated with increased pathological angiogenesis, evidenced by elevated levels of VEGF, TGF- $\beta$, FGF-21, TNFa, NO, and decreased levels of FLK-1 and sFLT1. Compared to glibenclamide, HESS in its highest dose played a weak role in preventing angiogenesis, but the combination of both partly enhanced the efficacy of the standard drug. Despite the high concentration of phenolic and flavonoid compounds in HESS, the weak anti-angiogenic effect may be due to the low applied concentration of the extract, or the presence of inhibitors in the composition of HESS. Additionally, no contradiction was observed between the herbal extract and the standard drug in combination thrapy. In future studies, it is recommended to use higher doses of HESS to observe its efficacy on blood levels of the aforementioned parameters as well as on tissues suffer from vascular disorders in diabetes. However, before treatment with higher doses, LD50 values, as well as the presence of interfering derivatives in the extract should be specified.

\section{Abbreviations}

S. securidaca: Securigera securidaca

HESS: hydroalcoholic extract of $S$. securidaca seed

G: glibenclamide 
VEGF: Vascular endothelial growth factor

FGF21: fibroblast growth factor 21

TGF- $\beta$ : transforming growth factor-beta

sFLT-1: soluble fms-like tyrosine kinase

FLK-1: fetal liver kinase 1

\section{Declarations}

\section{Acknowledgments}

This project was supported by Iran University of Medical Sciences with research No: 27813 . We sincerely thank the members of the Biochemistry Department of Iran University of Medical Sciences for their favors in improving this study.

\section{-Ethical Approval and Consent to participate}

Not applicable

\section{- Consent for publication}

Authors have read and approved the final version of the manuscript.

\section{- Availability of data and materials}

Data presented in this manuscript is available upon request

\section{- Competing interests}

The authors declare that they have no competing interests.

\section{- Funding}

This study was financially supported by Iran University of Medical Sciences

- Authors' contributions

All authors participated in project and article writing.

\section{- Acknowledgements}

Not applicable

\section{- Authors' information}


${ }^{1}$ Department of Biochemistry, Faculty of Medicine, Iran University of Medical Sciences, Tehran, Iran

${ }^{2}$ Department of Clinical Sciences, Faculty of Veterinary Sciences, Bu-Ali Sina University, Hamedan, Iran

\section{References}

1. Cade WT. Diabetes-related microvascular and macrovascular diseases in the physical therapy setting. Physical therapy. 2008;88(11):1322-35.

2. Krock BL, Skuli N, Simon MC. Hypoxia-induced angiogenesis: good and evil. Genes \& cancer. 2011;2(12):1117-33.

3. Krock BL, Skuli N, Simon MC. Hypoxia-induced angiogenesis: good and evil. Genes Cancer. 2011;2(12):1117-33.

4. Hickey MM, Simon MC. Regulation of angiogenesis by hypoxia and hypoxia-inducible factors. Current topics in developmental biology. 2006;76:217-57.

5. Ferrari G, Cook BD, Terushkin V, Pintucci G, Mignatti P. Transforming growth factor-beta 1 (TGFbeta1) induces angiogenesis through vascular endothelial growth factor (VEGF)-mediated apoptosis. Journal of cellular physiology. 2009;219(2):449-58.

6. Tomita Y, Ozawa N, Miwa Y, Ishida A, Ohta M, Tsubota K, et al. Pemafibrate Prevents Retinal Pathological Neovascularization by Increasing FGF21 Level in a Murine Oxygen-Induced Retinopathy Model. International journal of molecular sciences. 2019;20(23).

7. Chen S, Chen S-T, Sun Y, Xu Z, Wang Y, Yao S-Y, et al. Fibroblast growth factor 21 ameliorates neurodegeneration in rat and cellular models of Alzheimer's disease. Redox Biol. 2019;22:101133-

8. Alizadeh-Fanalou S, Babaei M, Hosseini A, Azadi N, Nazarizadeh A, Shojaii A, et al. Effects of Securigera Securidaca seed extract in combination with glibenclamide on antioxidant capacity, fibroblast growth factor 21 and insulin resistance in hyperglycemic rats. Journal of ethnopharmacology. 2020;248:112331.

9. Chen J-X, Stinnett A. Ang-1 gene therapy inhibits hypoxia-inducible factor-1alpha (HIF-1alpha)-prolyl4-hydroxylase-2, stabilizes HIF-1alpha expression, and normalizes immature vasculature in $\mathrm{db} / \mathrm{db}$ mice. Diabetes. 2008;57(12):3335-43.

10. Amano H, Kato S, Ito Y, Eshima K, Ogawa F, Takahashi R, et al. The Role of Vascular Endothelial Growth Factor Receptor-1 Signaling in the Recovery from Ischemia. PLoS One. 2015;10(7):e0131445e.

11. Sun Q, Heilmann J, König B. Natural phenolic metabolites with anti-angiogenic properties - a review from the chemical point of view. Beilstein J Org Chem. 2015;11:249-64.

12. Ataie A, Sabetkasaei M, Haghparast A, Moghaddam AH, Kazeminejad B. Neuroprotective effects of the polyphenolic antioxidant agent, Curcumin, against homocysteine-induced cognitive impairment and oxidative stress in the rat. Pharmacology, biochemistry, and behavior. 2010;96(4):378-85. 
13. Li A-N, Li S, Zhang Y-J, Xu X-R, Chen Y-M, Li H-B. Resources and biological activities of natural polyphenols. Nutrients. 2014;6(12):6020-47.

14. Parveen A, Subedi L, Kim HW, Khan Z, Zahra Z, Farooqi MQ, et al. Phytochemicals Targeting VEGF and VEGF-Related Multifactors as Anticancer Therapy. J Clin Med. 2019;8(3):350.

15. He Z, Chen AY, Rojanasakul Y, Rankin GO, Chen YC. Gallic acid, a phenolic compound, exerts antiangiogenic effects via the PTEN/AKT/HIF-1alpha/VEGF signaling pathway in ovarian cancer cells. Oncology reports. 2016;35(1):291-7.

16. Garjani A, Fathiazad F, Zakheri A, Akbari NA, Azarmie Y, Fakhrjoo A, et al. The effect of total extract of Securigera securidaca L. seeds on serum lipid profiles, antioxidant status, and vascular function in hypercholesterolemic rats. Journal of ethnopharmacology. 2009;126(3):525-32.

17. Rajaei Z, Hadjzadeh MA, Moradi R, Ghorbani A, Saghebi A. Antihyperglycemic and antihyperlipidemic effects of hydroalcoholic extract of Securigera securidaca seeds in streptozotocin-induced diabetic rats. Advanced biomedical research. 2015;4:33.

18. Singleton VL, Rossi JA. Colorimetry of total phenolics with phosphomolybdic-phosphotungstic acid reagents. American journal of Enology and Viticulture. 1965;16(3):144-58.

19. Chang $\mathrm{C}-\mathrm{C}$, Yang $\mathrm{M}-\mathrm{H}$, Wen H-M, Chern J-C. Estimation of total flavonoid content in propolis by two complementary colorimetric methods. Journal of food and drug analysis. 2002;10(3).

20. Kim JD, Kang SM, Seo BI, Choi HY, Choi HS, Ku SK. Anti-diabetic activity of SMK001, a poly herbal formula in streptozotocin induced diabetic rats: therapeutic study. Biol Pharm Bull. 2006;29(3):47782.

21. Bryan NS, Grisham MB. Methods to detect nitric oxide and its metabolites in biological samples. Free radical biology \& medicine. 2007;43(5):645-57.

22. Gupta N, Mansoor S, Sharma A, Sapkal A, Sheth J, Falatoonzadeh P, et al. Diabetic retinopathy and VEGF. The open ophthalmology journal. 2013;7:4-10.

23. Tsuchida K, Makita Z, Yamagishi S, Atsumi T, Miyoshi H, Obara S, et al. Suppression of transforming growth factor beta and vascular endothelial growth factor in diabetic nephropathy in rats by a novel advanced glycation end product inhibitor, OPB-9195. Diabetologia. 1999;42(5):579-88.

24. Krysiak O, Bretschneider A, Zhong E, Webb J, Hopp H, Verlohren S, et al. Soluble vascular endothelial growth factor receptor-1 (sFLT-1) mediates downregulation of FLT-1 and prevents activated neutrophils from women with preeclampsia from additional migration by VEGF. Circulation research. 2005;97(12):1253-61.

25. Cerezo AB, Winterbone MS, Moyle CW, Needs PW, Kroon PA. Molecular structure-function relationship of dietary polyphenols for inhibiting VEGF-induced VEGFR-2 activity. Molecular nutrition \& food research. 2015;59(11):2119-31.

26. Mirossay L, Varinská L, Mojžiš J. Antiangiogenic Effect of Flavonoids and Chalcones: An Update. Int J Mol Sci. 2017;19(1):27.

27. Park JJ, Hwang SJ, Park JH, Lee HJ. Chlorogenic acid inhibits hypoxia-induced angiogenesis via down-regulation of the HIF-1alpha/AKT pathway. Cellular oncology (Dordrecht). 2015;38(2):111-8. 
28. Cerezo AB, Hornedo-Ortega R, Álvarez-Fernández MA, Troncoso AM, García-Parrilla MC. Inhibition of VEGF-Induced VEGFR-2 Activation and HUVEC Migration by Melatonin and Other Bioactive Indolic Compounds. Nutrients. 2017;9(3):249.

29. Kimura T, Takagi H, Suzuma K, Kita M, Watanabe D, Yoshimura N. Comparisons between the beneficial effects of different sulphonylurea treatments on ischemia-induced retinal neovascularization. Free radical biology \& medicine. 2007;43(3):454-61.

30. Kuwabara M, Kakinuma Y, Ando M, Katare RG, Yamasaki F, Doi Y, et al. Nitric oxide stimulates vascular endothelial growth factor production in cardiomyocytes involved in angiogenesis. The journal of physiological sciences : JPS. 2006;56(1):95-101.

31. Maloney JP, Gao L. Proinflammatory Cytokines Increase Vascular Endothelial Growth Factor Expression in Alveolar Epithelial Cells. Mediators Inflamm. 2015;2015:387842-.

32. Jimenez V, Jambrina C, Casana E, Sacristan V, Munoz S, Darriba S, et al. FGF21 gene therapy as treatment for obesity and insulin resistance. EMBO molecular medicine. 2018;10(8).

33. Emanuelli B, Vienberg SG, Smyth G, Cheng C, Stanford KI, Arumugam M, et al. Interplay between FGF21 and insulin action in the liver regulates metabolism. J Clin Invest. 2014;124(2):515-27.

34. Lin S, Yu L, Ni Y, He L, Weng X, Lu X, et al. Fibroblast Growth Factor 21 Attenuates Diabetes-Induced Renal Fibrosis by Negatively Regulating TGF- $\beta$-p53-Smad2/3-Mediated Epithelial-to-Mesenchymal Transition via Activation of AKT. Diabetes Metab J. 2020;44(1):158-72.

35. Fu Z, Gong Y, Liegl R, Wang Z, Liu CH, Meng SS, et al. FGF21 Administration Suppresses Retinal and Choroidal Neovascularization in Mice. Cell reports. 2017;18(7):1606-13.

\section{Figures}

Comparison of Insulin levels among study groups

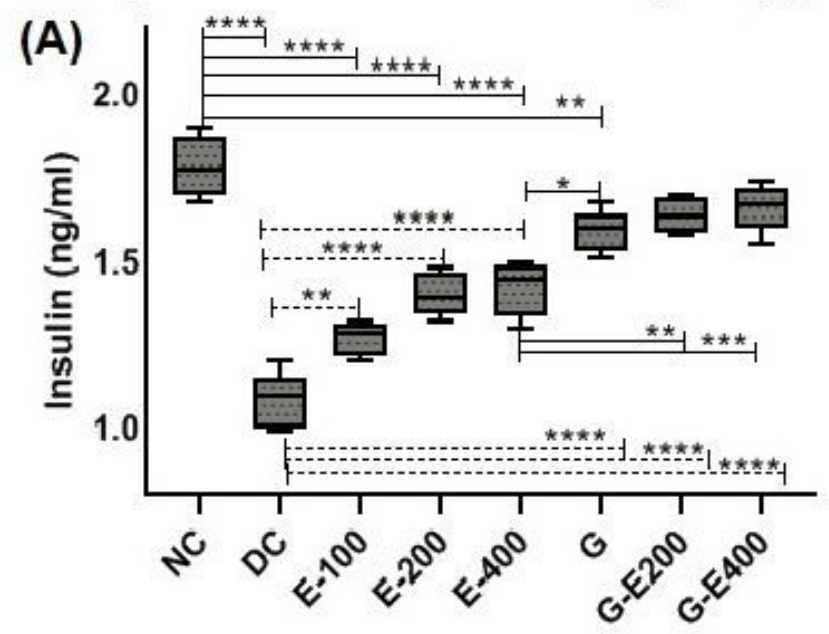

Groups
Comparison of glocose levels among study groups

(B)

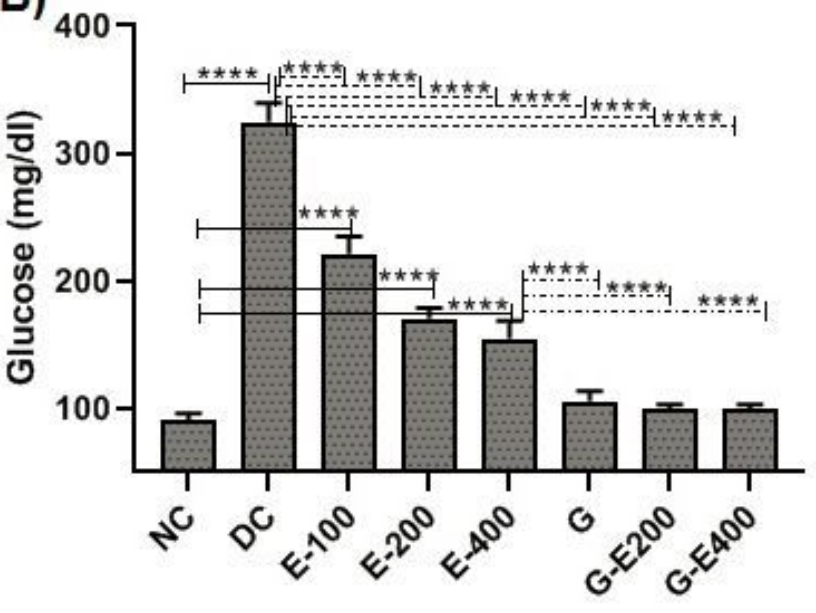

Groups

Figure 1 
Comparison of Insulin (A) and glucose (B) levels among experimental and control groups. HESS (hydroalcoholic extract of S. securidaca seeds); G (glibenclamide); DC (Diabetic control); NC (normal control. (Values are means \pm SD. * Significant difference degree among groups.

(A) Comparison of VEGF levels among study groups

(B) Comparison of SFLT-1 levels among study groups
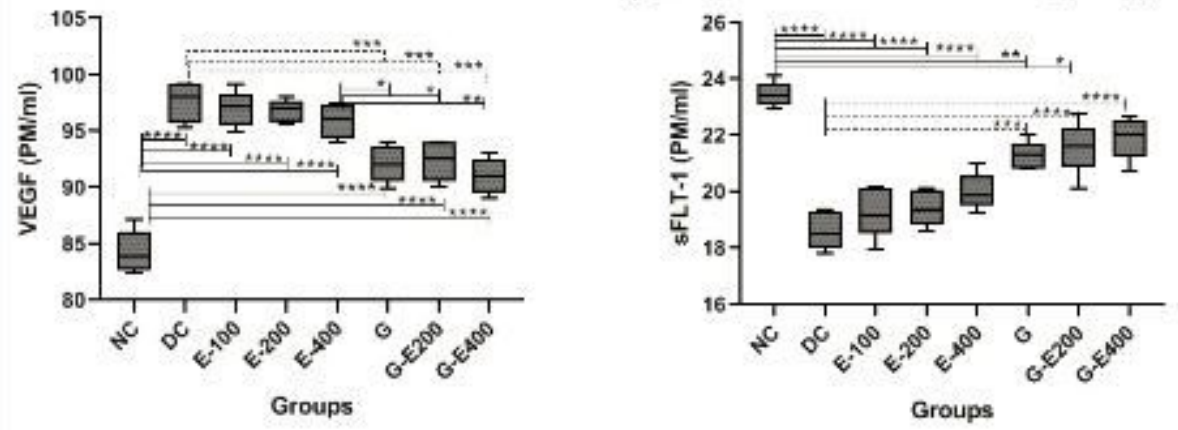

(C) Comparison of FLK1 levels among study groups

(D) Comparison of FGF21 levels among study groups
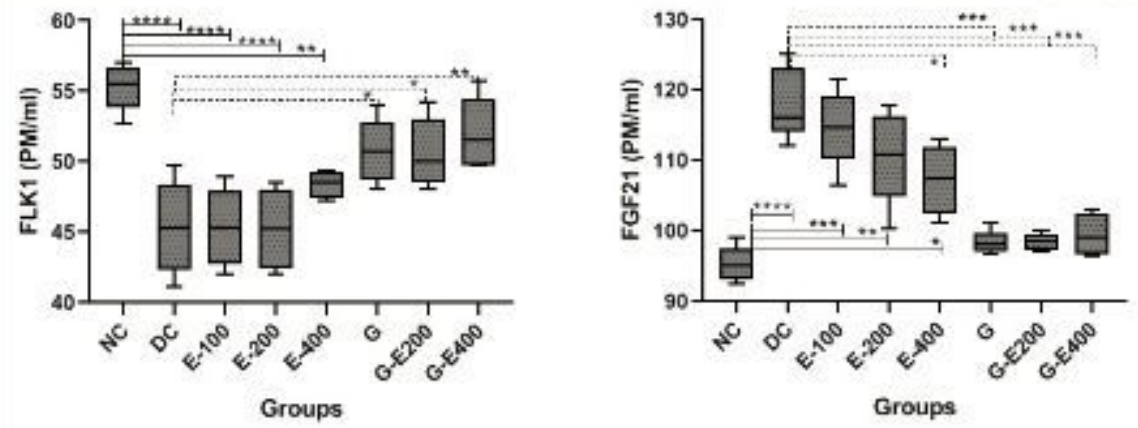

(E) Comparison of TGF-B levels among study groups
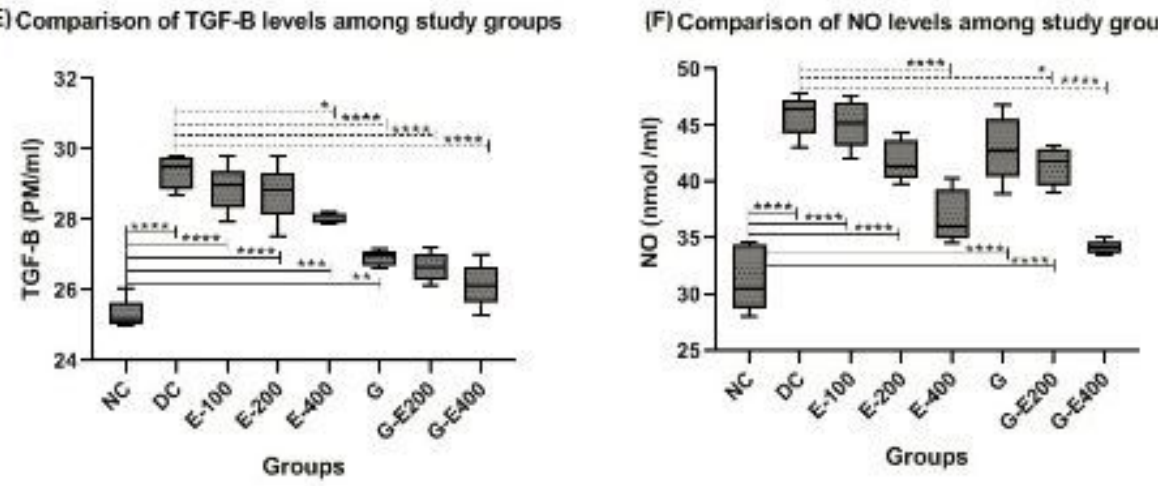

Figure 2

comparison of pro/ anti-angiogenic factors levels among experimental and control groups. (A) VEGF (Vascular endothelial growth factor), (B) sFLT-1 (soluble fms-like tyrosine kinase), (C) FLK-1 (fetal liver kinase 1), (D) FGF21 (fibroblast growth factor 21), (E) TGF- $\beta$ (transforming growth factor-beta), (F) NO (nitric oxide). HESS (hydroalcoholic extract of S. securidaca seeds); G (glibenclamide); DC (Diabetic control); NC (normal control.( Values are means \pm SD. * Significant difference degree among groups. 